\title{
THE USE OF THE NON-CONTACTING TEMPERATURE MEASURING TECHNIQUES IN THE EARLY DETECTION OF HIDDEN FAULTS IN ELECTRONIC COMPONENTS ${ }^{\dagger}$
}

\author{
P. TRÄGNER \\ Technical University, Dresden, GDR \\ (Received November 1, 1982; in final form July 12, 1983)
}

\begin{abstract}
One of the reasons for a reduction of production quality is the so-called 'hidden faults', i.e. defects which will not be detected by the available testing equipment in spite of measures for ensuring a good quality. These testing methods, which are mostly electrical, can be supplemented in a suitable way by non-contacting temperature measuring techniques, since the variation of temperature as a function of operating conditions and environment constitutes one of the most important problems in the design of electronic components and sub-assemblies, and of their reliability.

In this paper a relation is presented between a qualitative diagnostic model, reserve stability and other characteristics, on the one hand, and the permissible thermal energy that can be radiated, on the other hand, which facilitates the detection of hidden faults.
\end{abstract}

\section{INTRODUCTION}

At all times the securing of high quality stable industrial products is one of industry's essential tasks. One of the reasons for a reduction of production quality is the existence of so-called hidden faults, i.e. defects which in a practical situation are not initially taken into account by the measures and procedures used for ensuring good quality. A large proportion of such faults occur within the first few hours of operation. However, a large number of failures due to hidden faults also occur after considerable operating times. To a certain extent the application of non-destructive testing procedures allows one to detect deviations in the properties of electronic components and thus to determine in advance the reliability to be expected. Such testing can be done on a real time or an accelerated time basis, and avoids the necessity of having to wait for quantitative data on the frequency of failure and the failure modes themselves being forwarded from the user. Non-destructive methods of reliability characteristic determination can give useful information on reliability, providing the testing conditions are fully specified, and that the data is obtained by the manufacturer only after considerable time delay.

Due to the variety and diversity of the materials to be processed and the complexity of the production technology associated with components and subassemblies, complex testing procedures and methods have become necessary and new variants of defect analysis are continually being investigated. A rational combination of destructive and non-destructive testing techniques together with the systematic selection of the testing devices and procedures require an overall knowledge of the testing characteristics. In the majority of cases these testing characteristics are not well known, although in certain circumstances, e.g. the relationship between operating temperature and service ability, more information is available.

\footnotetext{
${ }^{\dagger}$ Original version of paper originally given at the 5th International Spring Seminar on Electrotechnology, Prenet, Czechoslovakia, 1-4 June, 1982.
} 
In many cases the so-called hidden faults do not manifest themselves in a change of the characteristic observed by the electrical testing device. However, since they often cause a change in temperature of the individual component or of the temperature field over the component, temperature measurement is often a useful way of determining the possibility for potential failure in a component. ${ }^{1}$

\section{PRINCIPLES OF ANALYSIS}

In electronic devices the conversion of energy into heat will depend on the characteristic values of the components and on the existence of defects, given that all other conditions remain constant. The temperature field observed will be dependent on the arrangement of the components as well as the conversion of energy to heat in the components themselves. In most cases a change in the temperature of individual components in a subassembly will cause a change of the overall thermal state of the subassembly itself. Thus, by checking through the temperature field it is possible to eliminate components that have parameters outside of specified limits. The existence of a defect in a component may cause the component to run at an elevated or at too low a temperature compared with a device that is operating satisfactorily. The derivation of a standard of comparison of temperature from a mathematical model is possible, but is complicated, and at present such an analysis is available only for selected components, special arrangements, or specific manufacturing processes. In some cases if the expenditure required for the analysis is to be kept within reasonable limits, approximations will have to be made which will in turn cause deviations in the model and can render the whole analysis inapplicable. However, experimental derivation of a comparative standard from a flawless specimen, and the application of this to the analysis of components, often by automatic means, can be less expensive. Generally, the temperature field is determined from the temperature values of the individual components or groups of components. The temperature field results only from the interaction between the individual components in the subassembly. Therefore, the use of noncontacting temperature testing methods is effective for the purpose of reliability prediction. Furthermore, it should be noted that the temperatures observed will be influenced by the thermal testing procedure used; the actual existing temperature conditions will be influenced least by the non-contacting temperature measuring techniques.

The existence of hidden faults in components from a production run can sometimes be determined by the analysis of the characteristic features which would result from such faults, e.g. changes in dimension, colour, electrical properties, mechanical properties, etc. The probability of the occurrence of such faults will be related to the fault mechanism. However, such an analysis requires the assembly of a large amount of statistical data, and although it is recognized that this is the best approach, information will be lacking during the development of devices and start of a production run. In such a situation a possible solution is provided by an experimentally based computational evaluation using a diagnostic model. This paper is concerned with the development of such a diagnostic model with particular reference to its applicability to temperature observations.

\section{DIAGNOSTIC MODEL}

\subsection{General}

In a qualitative diagnostic model ${ }^{2}$ there should be recordable $\mathrm{i}(1, \mathrm{~m})$ defects. For each of these faults or defects a characteristic quantity $x_{i}$ can be defined as typical and the distribution function and dependence on time of this characteristic quantity is known. 
If the number of faults is $n_{i}\left(t, x_{i}\right)$ and the probability of fault detection by the testing device is $p_{i, j}\left(x_{i}\right)$ and the probability that a fault will lead to a failure is $\sigma_{i}\left(x_{i}\right)$, then although a check has been made the number of faulty products, qaus, can be written as

$$
q_{\text {aus }}(t)=\sum_{j=1}^{1} \sum_{i=1}^{m} \frac{\int_{x_{i}=0}^{\infty} n_{j}\left(t, x_{i}\right)\left[1-p_{i, j}\left(x_{i}\right)\right] \sigma_{i}\left(x_{i}\right) d x_{i}}{\int_{x_{j}=0}^{\infty} n_{i}\left(t, x_{i}\right) d x_{i}} .
$$

No testing is done, $\mathrm{p}_{\mathrm{ij}}$ will be equal to 0 .

It is necessary in using equation (1) to choose that quantity for examination for which the function $\sigma_{\mathrm{i}}\left(\mathrm{x}_{\mathrm{i}}\right)$ has the highest sensitivity. For example, in the case of a soldered joint in which the fault has been determined by the indirectly tested parameter, "void", a typical characteristic quantity which would be used would be the maximum permissible diameter of the void. (i.e., $x_{i}$ would be equal to $d_{\text {max }}$ ).

In order to judge the technical state of a product with the aid of non-destructive test procedures it is important to know the physical-chemical processes which would lead to failure. If these are known then it is possible to detect those kinetic processes which influence the formation of a specific mechanism for failure and contribute to it, and it should therefore be possible to determine the most useful characteristic quantity for observation. If the characteristic parameter used is that of temperature based on knowledge of failure mechanisms, then in addition to the statistical specification of temperature a certain reserve stability needs to be taken into account.

In order to calculate the probability, ${ }^{3} \mathrm{q}$, of the occurrence of a failure, one must take into account the reduction and rate of change of the reserve stability (mechanical, electrical, thermal), q will then be given by

$$
q=\int_{0}^{t} \frac{\partial q}{\partial t} d t=\int_{0}^{t} \frac{\partial q}{\partial(\Delta x)} \frac{\partial(\Delta x)}{\partial t} d t,
$$

where $\Delta x$ - reserve stability

$\partial(\Delta x) / \partial \mathrm{t}$ - rate of change of the reserve stability as a result of various processes in the material

$\partial q / \partial(\Delta x)-$ probable state of stability of the material.

As a rule, a number $\mathrm{k}$, of the characteristic quantities will contribute to the stability.

Thus one can write

$$
\Delta \mathrm{x}=\sum_{i=1}^{\mathrm{k}} \Delta \mathrm{x}_{\mathrm{i}}
$$

Thus

$$
q=\int_{0}^{t} \frac{\partial q}{\partial(\Delta x)}\left[\sum_{i=1}^{k} \frac{\partial\left(\Delta x_{i}\right)}{\partial t}\right] d t
$$

Equation (4) shows that the probability of the failure grows with the reduction of the 
reserve stability. A stability reserve manifests itself, e.g., during the employment of a capacitor, in the difference value between its actual operating voltage and its limiting load voltage. For the use of capacitors in portable colour television sets this difference value is frequently lower since smaller dimensions of the components are foremost, which is less important for large-size, non-portable sets. Since, in addition, the ventilating conditions are more unfavourable compared with non-portable sets, failures will occur more frequently.

\subsection{Temperature Model}

If the characteristic quantity being measured in a non-contacting, non-destructive sense is that of temperature, the parameters measured on a pyrometer will consist of two components: These are:-

Radiation from the measured spot and background radiation or reflected radiation.

Thus one can write for the energy output from the pyrometer that the energy $W_{p}$ is given by

$$
\mathrm{W}_{\mathrm{p}}=\mathrm{A}\left[\varepsilon \mathrm{T}^{4}+(1-\varepsilon) \mathrm{T}_{\mathrm{H}^{4}}\right] .
$$

where:-

A - device constant

$\varepsilon-$ radiation coefficient of the surface to be examined (measured)

$\mathrm{T}$ - temperature of the measured spot

$\mathrm{T}_{\mathrm{H}}-$ temperature of the background

The temperature of the measured spot, $\mathrm{T}$, will be obtained from the sum of two temperatures, namely the environmental temperature, $\mathrm{T}_{\mathrm{U}}$, associated with the undisturbed sections of the specimen, and the heating processes due to the presence of heat sources, $\Delta \mathrm{T}$, resulting from faults in the component or groups of components, i.e., one can write

$$
\mathrm{T}=\mathrm{T}_{\mathrm{U}}+\Delta \mathrm{T}
$$

Applying equation 6 to equation 5 one obtains

$$
\mathrm{W}_{\mathrm{p}}=\mathrm{A}\left\{\varepsilon \mathrm{T}_{\mathrm{U}}^{4}\left[1+4\left(\frac{\Delta \mathrm{T}}{\mathrm{T}_{\mathrm{U}}}\right)+6\left(\frac{\Delta \mathrm{T}}{\mathrm{T}_{\mathrm{U}}}\right)^{2}+4\left(\frac{\Delta \mathrm{T}}{\mathrm{T}_{\mathrm{U}}}\right)^{3}+\left(\frac{\Delta \mathrm{T}}{\mathrm{T}_{\mathrm{U}}}\right)^{4}\right]+(1-\varepsilon) \mathrm{T}_{\mathrm{H}}^{4}\right\} .
$$

In terms of the temperature change due to heat sources arising from faults, $\Delta T$, the product for $4 \mathrm{~A} \varepsilon \Delta \mathrm{TT}^{3} \mathrm{U}$ will be the most important. Thus equation (7) may be approximated as follows

$$
\mathrm{W}_{\mathrm{p}} \approx \mathrm{A}\left[\varepsilon \mathrm{T}_{\mathrm{U}}^{4}\left(1+4 \frac{\Delta \mathrm{T}}{\mathrm{T}_{\mathrm{U}}}\right)+(1-\varepsilon) \mathrm{T}_{\mathrm{H}}^{4}\right]
$$

In the case of fault-free specimens, $\Delta \mathrm{T}$ will be equal to 0 , and in this case equation (8) becomes

$$
\mathrm{W}_{0}=\mathrm{A}\left[\varepsilon \mathrm{T}_{\mathrm{U}^{4}}+(1-\varepsilon) \mathrm{T}_{\mathrm{H}^{4}}\right.
$$

Thus, the change in pyrometer output, $\Delta \mathrm{W}_{\mathrm{p}}$ due to faults in the components can be written as

$$
\Delta \mathrm{W}_{\mathrm{p}}=4 \mathrm{A \varepsilon T}_{\mathrm{U}}^{3} \Delta \mathrm{T}
$$


Approximations cause errors which are found to be not greater than $1 \%$ within the range of the application of non-contacting temperature measuring techniques. This situation can be mathematically described in terms of the relative error, $\delta$, such that

$$
\delta=\frac{\mathrm{W}_{\mathrm{p}}-\mathrm{W}_{\mathbf{0}}-\Delta \mathrm{W}_{\mathrm{p}}}{\mathrm{W}_{\mathrm{p}}-\mathrm{W}_{0}}
$$

\section{DISCUSSION AND CONCLUSIONS}

If the range of hidden faults gives an excess temperature of about $30 \%,-$ any larger excess temperatures would be associated with observable faults, - then the relative error, $\delta$, which is a function of $\Delta \mathrm{T}$ will remain below that given by $\delta=0.47 \Delta \mathrm{T}(\%)$.

At the present level of engineering, however, errors due to optical pyrometer measurements of $\pm 1 \%$ or more cannot be excluded. However, the use of microcomputers in pyrometry not only allows for these errors, but also for the examination of failures in which high excess temperatures are observed. These may occur at inaccessible locations if components are used in heavy current engineering and power electronics. Errors, however, can be reduced considerably if the temperature of the hot spot is compared with that of the local neighbourhood and is not determined in absolute terms.

The need for the non-destructive testing of components in order to detect hidden faults has been discussed and a diagnostic model for examining components for faults has been derived. The specific application of the model to the case where the characteristic parameter examined is the surface temperature of the component has been considered, and it has been shown that this technique can be used for detecting hidden faults that produce the changes in temperature. The technique can be improved if comparative temperatures are measured so that temperature differences between the hot spot and the local neighbourhood are taken as the characteristic quantity.

The temperature measuring devices used for the investigations allow a temperature resolution of $1 \mathrm{~K}$ or $0.5 \mathrm{~K}$, resp., and have shown that infrared radiometry can be applied for the indirect and non-contacting measurement of the local temperature and for the detection of reliability errors. Such investigations were carried out on ohmic resistances and contacts; ${ }^{4}$ in the dimensioning of hybrid circuits $;{ }^{5}$ in the quality control for plated through holes; ${ }^{6}$ for the sorting of toroidal cores for memory matrices, ${ }^{7}$ and with respect to the designing of manufacturing and testing processes they supplied essential information, which has contributed to reducing the number of hidden faults and to improving reliability.

\section{REFERENCES}

1. L. Walther, D. Gerber, "Infrarotmesstechnik", Berlin (1981), Verlag Technik.

2. B.E. Beredichevski, "Non-destructive Testing of Units and Components of Electronic Devices." Moscow 1976, Radio (in Russian). pp. 238-246.

3. N.S. Danilin, "Non-destructive Testing of Production Quality in Electronics." Moscow 1976, Standards edition (in Russian).

4. H. Stojanoff, "Ursachen und Einflüsse von Fehlern bei Temperaturmessungen an bestückten Leiterplatten" Dissertation of the Technical University of Dresden (1973).

5. M. Wetzko, P. Streubel, I. Althus. "Universeller Frequenzteiler in Hybridtechnik." Radio fernsehen elektronik, 28, 1, pp. 19-21, (1979).

6. H. Helm, "Infrarotprüfung von Durchkontaktierungen." Feingerätetechnik, 22, 10, pp. 444-446 (1973).

7. E. Feige, W. Jung.: "Ein Beitrag zur Entwicklung von Messeinrichtungen zur Prüfung von Ferritkernen und Ferritkernmatrizen durch Ausnutzung der Infrarotstrahlung." Dissertation of the Technical University of Dresden (1976). 

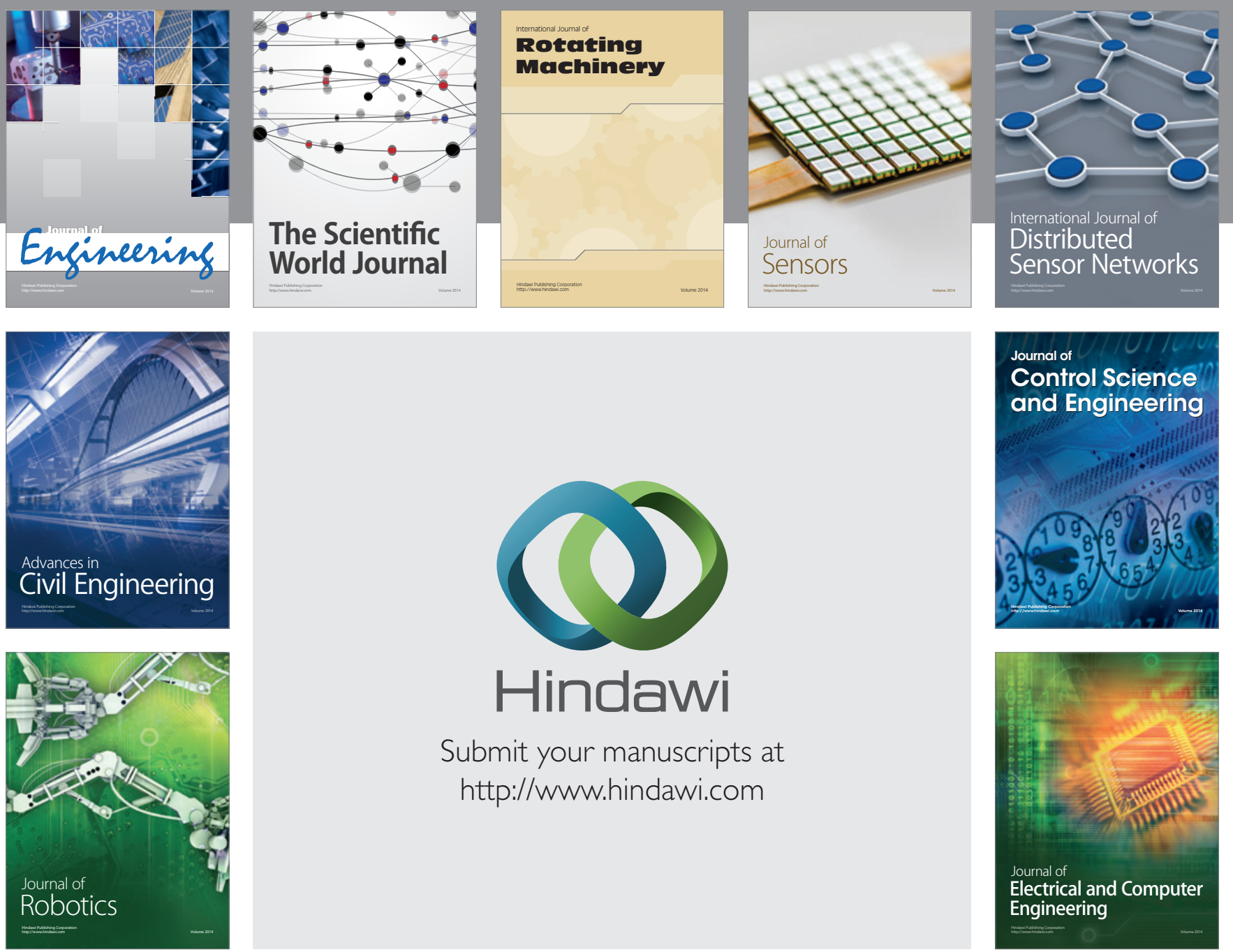

Submit your manuscripts at

http://www.hindawi.com
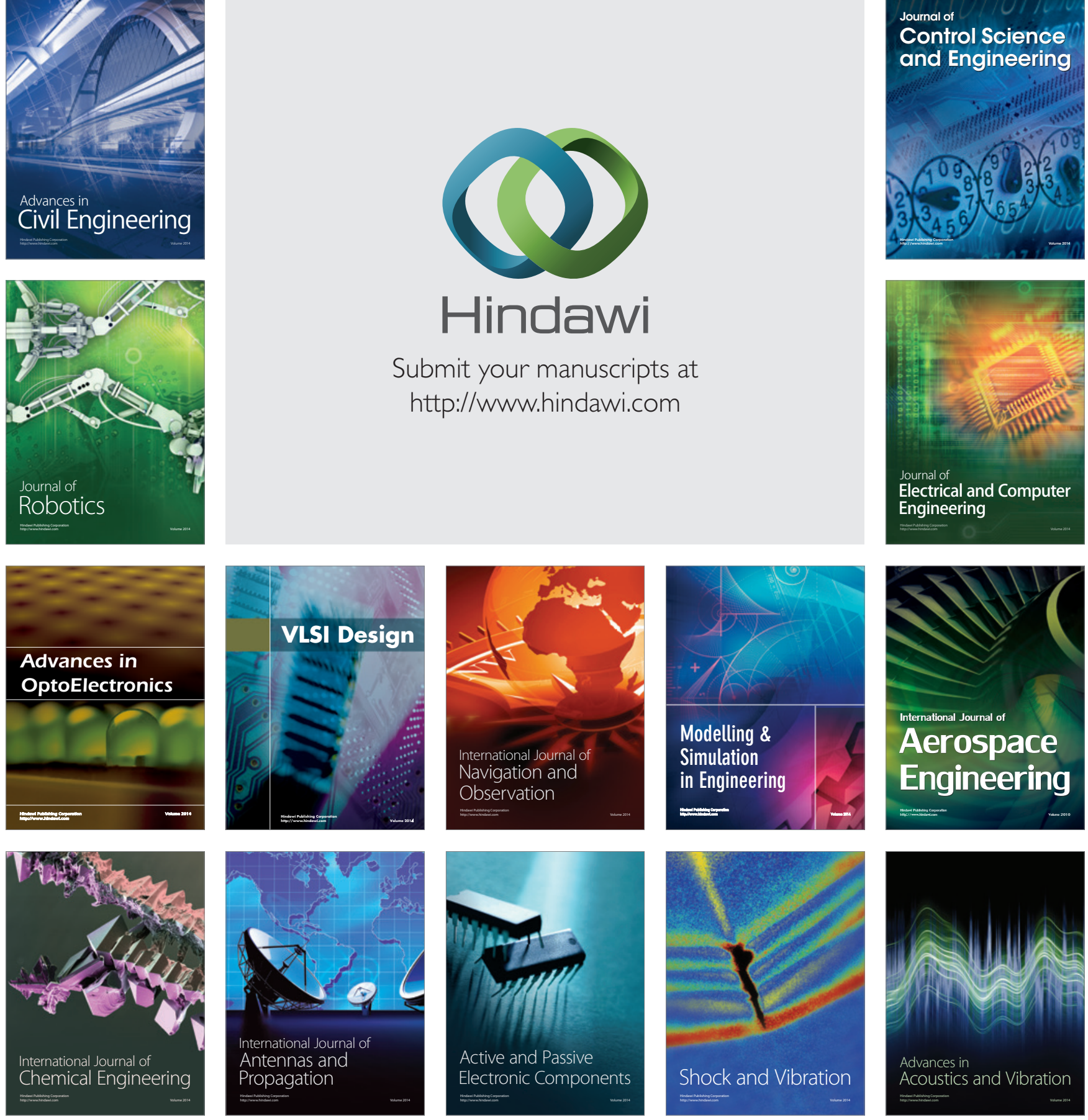